\title{
Anti-inflammatory effects of free and liposome-encapsulated natural Algerian thermal water in RAW 264.7 macrophages
}

\author{
Romaissaa Mokdad $^{\mathrm{a}, *}$, Cendrine Seguin ${ }^{\mathrm{b}}$, Sylvie Fournel ${ }^{\mathrm{b}}$, Benoît Frisch ${ }^{\mathrm{b}}$, Béatrice Heurtault ${ }^{\mathrm{b}, *}$, \\ Abdelkader Hadjsadok ${ }^{\mathrm{a}, *}$ \\ a Laboratoire de l'analyse fonctionnelle des procédés chimiques, Département de génie des \\ procédés, Faculté de Technologie, Université de Blida 1, 270 route de Soumaa, 09000 Blida, \\ Algérie \\ b 3BIO Team, UMR 7199, Université de Strasbourg/CNRS, Faculté de Pharmacie, 74 route du \\ Rhin, 67401 Illkirch Cedex, France \\ * Corresponding authors. \\ E-mail addresses: mokdadromaissa@univ-blida.dz (R. Mokdad), bheurtault@unistra.fr (B. \\ Heurtault), hadjsadok@yahoo.fr (A. Hadjsadok)
}

\begin{abstract}
The main objectives of this work were to formulate liposomes encapsulating highly mineralized thermal waters (TWs) and to study the potential anti-inflammatory effect of free and encapsulated thermal waters on RAW 264.7 macrophage cells stimulated with lipopolysaccharide (LPS). TWsloaded conventional and deformable liposomes (TWs-Lip and TWs-DLip) were prepared by sonication and extrusion, respectively. They were characterized for their vesicle size, zeta potential, entrapment efficiency, physical stability and in vitro anti-inflammatory effect. Formulated suspensions have a low polydispersity and nanometric size range with zeta potential values closed to zero. The vesicle size was stable for 30 days. Entrapment efficiency of TWs in conventional and deformable liposomes was found to be higher than $90 \%$ and $70 \%$, respectively. Pretreatment of LPS-stimulated murine macrophages with TWs resulted in a significant reduction in NO production and modulated TNF- $\alpha$ production suggesting an anti-inflammatory effect which were even more striking with TWs-Lip and TWs-DLip. Liposome formulations may offer a suitable approach for transdermal delivery of TWs indicated in inflammatory skin diseases.
\end{abstract}

Keywords: Algerian thermal waters, Liposomes, Anti-inflammatory effect, Nitric oxide, Cytokine.

\section{Introduction}

Skin forms the first barrier and line of immunological defense against pathogens and noxious substances. Extensive crosstalk between epithelial, stromal and immune cells regulates immune responses in the skin to ensure effective host defense and to maintain or restore tissue homeostasis. However, dysregulated of these equilibrium can cause chronic inflammatory skin diseases such as psoriasis, atopic dermatitis, and hidradenitis suppurativa (Pasparakis, Haase et al. 2014). These disorders result in significant morbidity and adversely affect patients quality of life (Merial-Kieny, Mengual et al. 2011). Immune cell populations in the skin are predominantly composed of dendritic cells (DCs) and macrophages (Malissen, Tamoutounour et al. 2014). 
Resident skin macrophages are thought to survey the tissue and carry out the early detection of, and response to, antigen entering the body through the skin (Pasparakis, Haase et al. 2014).

The inflammatory response leads to the production of inflammatory mediators including nitric oxide (NO) and inflammatory cytokines (such as tumor necrosis factor- $\alpha$ (TNF- $\alpha$ )). NO is a reactive radical molecule synthesized by a family of enzymes called nitric oxide synthases (NOS) from L-arginine, $\mathrm{O}_{2}$ and NADPH (Nathan and Xie 1994). In macrophages, the inducible NOS isoform (iNOS or NOS-2) produces a huge amount of $\mathrm{NO}$ as a host defense response to pathogens (Xie, Cho et al. 1992, MacMicking, Xie et al. 1997). TNF- $\alpha$ is an important mediator of inflammation, produced and secreted by a variety of cell types, principally by monocytes and macrophages (Liu, Sidiropoulos et al. 2000). But, overproduced, it contributes to the pathogenesis of a variety of skin conditions such as in psoriatic lesions (Dal Pra, Chiarini et al. 2007). Thus, equilibrium between a necessary defense response and too much response is not that easy to find.

Thermal waters (TWs) are natural mineral waters formed under specific geological conditions, and characterized by a rich composition with chemical and physical elements (Ghersetich and Lotti 1996). Over the past decades, the use of thermal waters is gaining popularity in dermatologic practice. They have been extensively used in several skin diseases, such as atopic dermatitis and skin dryness (Nasermoaddeli and Kagamimori 2005). The use of these waters for therapeutic purposes is based on their composition. To date, the mechanisms involved are only partially understood (Nasermoaddeli and Kagamimori 2005). In vitro experiments have demonstrated the effect of different thermal waters, in regard to their composition, on membrane fluidity, their antiradical and anti-inflammatory properties, their effects on many mediators involved in the immune response and their stimulating effect on keratinocyte differentiation (Merial-Kieny, Castex-Rizzi et al. 2011, Seite 2013, Tacheau, Weisgerber et al. 2018). Numerous studies have also used cell culture to determine the molecular mechanisms of TWs action at cutaneous level (Dal Pra, Chiarini et al. 2007, Joly, Branka et al. 2014, Karagülle, Karagülle et al. 2018). Furthermore, thermal waters were successfully included as raw materials for dermocosmetic formulations to achieve the cutaneous welfare (Almeida, Madeira et al. 2019, Nunes, Rodrigues et al. 2019, Tan, Spada et al. 2020).

Colloidal lipid-based vesicles, such as liposomes, have shown promising results as drug delivery systems through transdermal route. Liposomes are spherical vesicles, composed of lipid bilayer that encapsulates aqueous phase. This amphiphilic structure allows the incorporation of watersoluble materials into the aqueous core (Wang, Swing et al. 2020). Recent approaches have resulted in the design of deformable liposomes and ethosomes able to improve skin delivery of various drugs, consisted of phospholipids and an edge activator and ethanol, respectively (Elsayed, Abdallah et al. 2006). Further, liposomes were found to deliver efficiently the hydrophilic substances to the deeper skin tissues in higher concentrations than conventional dosage forms, while providing prolonged release (Verma, Verma et al. 2003, Betz, Aeppli et al. 2005). The encapsulation of TWs in liposomes seems to be an interesting alternative to improve 
their skin permeation (Araujo, Paiva et al. 2015), as long as the percutaneous penetration of water increased when the stratum corneum is damaged and decreased when it is healthy (Blattner, Coman et al. 2014, Carbajo and Maraver 2018). To date, little scientific works have been published on this subject. Liposomes based on phospholipids and cholesterol, encapsulating an oligomineral TW and stabilized in a DNA gel were proposed, in a published patent in 1992, for a cosmetic and dermatologic applications (Fabre, Cousse et al. 1992). An in-vitro study has recorded that TW-encapsulating liposomes can penetrate through the skin barrier until they reached the spinous layer; these formulations have proved an effective moisturizing and antiinflammatory effects on keratinocyte cells (Nunes and Tamura 2012). Moreover, stable deformable niosomes formulated with surfactants and cholesterol and charged with a TW have increased its cutaneous penetration and consequently increased the skin hydration and mineralization (Manosroi, Witkittilak et al. 2010).

In this work, we were led to encapsulate Algerian highly mineralized thermal waters in conventional and deformable liposomes, investigate their physicochemical characteristics and compare their anti-inflammatory effect to that of non-encapsulated TWs. The anti-inflammatory potential of TWs and liposomal formulations loaded with TWs was carried out in an in vitro model, the RAW 264.7 murine macrophage cell line activated by LPS, a molecule that mimics activation by bacteria.

\section{Abbreviations:}

$A D$, atopic dermatitis;

BTW, Baraka thermal water;

$\mathrm{CH}$, cholesterol;

DLip, deformable liposomes;

DLS, dynamic light scattering;

DMSO, dimethyl sulfoxide;

Lip, liposomes;

LPS, lipopolysaccharide;

$M L V$, multilamellar vesicles;

$P B S$, phosphate buffer saline;

$P C$, phosphatidylcholine;

$P G$, phosphatidyl glycerol;

$P d I$, polydispersity index;

RAW 264.7, Ralph And William's cell line 264.7;

ROS, Reactive oxygen species;

STW, Salhine thermal water;

SDC, sodium deoxycholate;

$T_{m}$, transition temperature;

$T N F$, tumor necrosis factor;

TWs, thermal waters 


\section{Materials and methods}

\subsection{Reagents}

\subsubsection{Chemical reagents}

Soybean L- $\alpha$-phosphatidylcholine (PC, 99\% purity) was purchased from Sigma GmbH Chemical Company (USA). Cholesterol $(\mathrm{CH})$ was provided from Sigma-Aldrich (St. Quentin Fallavier, France) and recrystallized in methanol in the laboratory. Egg yolk L- $\alpha$-phosphatidyl-DL-glycerol (PG, 99\% purity) was purchased from Avanti Polar Lipids, Inc. (Albama, USA). Stock solutions of PC (25 mM), PG (25 mM) and $\mathrm{CH}(25 \mathrm{mM})$ were prepared in chloroform/methanol (9/1 (v/v)) and conserved under argon atmosphere at $-20{ }^{\circ} \mathrm{C}$. Sodium deoxycholate (SDC, $97 \%$ purity) was purchased from Sigma-Aldrich. Spectra/Pore® dialysis membrane (12,000-14,000 molecular weight cut off) was purchased from Spectrum Laboratories, Inc. (USA). Griess reagents were purchased from Sigma (St. Louis, MO, USA). All solvents used were of analytical or HPLC grade or equivalent. All other reagents were commercially available and used without further purifications. Purified $18 \mathrm{M} \Omega$ ultrapure-water (Elga, Veolia, UK) was used to prepare reagent solutions.

\subsubsection{Biological reagents}

Dulbecco's Modified Eagle's Medium (DMEM), heat-inactivated fetal bovine serum (FBS), penicillin and streptomycin, phosphate buffered saline (PBS, pH 7.4), and other supplements for cell culture were purchased from Sigma Aldrich (Saint Louis, MO). Lipopolysaccharide (LPS) extracted from Escherichia coli (K12) was purchased from Invivogen (San Diego, CA). Purified anti-mouse TNF- $\alpha$ antibody clone 1F3F3D4 and biotinylated anti-mouse TNF- $\alpha$ antibody clone XT3/XT22 for ELISA testing were purchased from eBioscience/ThermoFischer Scientific (Waltham, MA). Horseradish Peroxidase Avidin (Avidin HRP) was from Jackson (West Grove, PA).

\subsection{Thermal waters}

TWs were taken from two thermal springs located in the region of Biskra, southeast of Algeria: Baraka (BTW) and Salhine (STW). BTW and STW samples were collected directly from the springs, transported refrigerated to the laboratory and then filtered with a Millipore $0.22 \mu \mathrm{m}$ vacuum-driven disposable filtration Stericup (Millipore, Billerica, MA) to remove any microorganisms and sand. Later, they were stocked in glass bottles at $4{ }^{\circ} \mathrm{C}$ until uses. Prior to the analysis of minor elements ( $\mathrm{Fe}, \mathrm{Zn}, \mathrm{Mn}, \mathrm{Cu}, \mathrm{Se}$ ), water samples were acidified with $0.5 \%$ (v/v) concentrated $\mathrm{HNO}_{3}$ and measured by inductively coupled plasma - atomic emission spectrometry (ICP-AES). The other physico-chemical parameters were completed by detailed information

published in previous works published by Chenaker et al. (Chenaker, Houha et al. 2018). Thermal water osmolality was measured in triplicate using a freezing point osmometer (Advanced Instruments Model 3D3, Norwood, MA). Physiochemical composition and osmolality of BTW and STW are summarized in Table 1. 


\section{Table 1}

Chemical analysis of the used thermal waters

\begin{tabular}{llll}
\hline & & $\mathrm{STW}$ & $\mathrm{BTW}$ \\
\hline Temperature $\left({ }^{\circ} \mathrm{C}\right)$ & & $\mathbf{4 5}$ & $\mathbf{4 2}$ \\
pH & & 7.2 & $\mathbf{6 . 9}$ \\
Conductivity $(\mu \mathrm{S} / \mathrm{cm})$ at $\mathrm{25}^{\circ} \mathrm{C}$ & & $\mathbf{1 4 7 8 0}$ & $\mathbf{4 4 1 0}$ \\
Calcium & $\mathrm{Ca}^{2+}$ & $\mathbf{3 3 9 . 4 6}$ & $\mathbf{4 3 4 . 2 3}$ \\
Magnesium & $\mathrm{Mg}^{2+}$ & $\mathbf{4 5 . 5 6}$ & $\mathbf{7 9 . 5 4}$ \\
Sodium & $\mathrm{Na}^{+}$ & $\mathbf{2 9 3 6 . 6 5}$ & $\mathbf{4 6 1 . 7 6}$ \\
Potassium & $\mathrm{K}^{+}$ & $\mathbf{7 9 . 5 8}$ & $\mathbf{4 3 . 7 5}$ \\
Chloride & $\mathrm{Cl}^{-}$ & $\mathbf{4 5 1 2 . 4 3}$ & $\mathbf{5 3 8 . 6 7}$ \\
Sulfate & $\mathrm{SO}_{4}{ }^{2-}$ & $\mathbf{1 4 8 5 . 0 9}$ & $\mathbf{1 7 4 2 . 9 2}$ \\
Bicarbonates & $\mathrm{HCO}_{3}{ }^{-}$ & $\mathbf{3 8 6 . 7 4}$ & $\mathbf{1 3 4 . 2 0}$ \\
Fluoride & $\mathrm{F}^{-}$ & $\mathbf{1 . 7 5}$ & $\mathbf{0 . 6 6}$ \\
Bromide & $\mathrm{Br}^{-}$ & $\mathbf{5 . 6 9}$ & $\mathbf{1 . 7 8}$ \\
Nitrate & $\mathrm{NO}_{3}{ }^{-}$ & $\mathbf{N D}$ & $\mathbf{0 . 5 5}$ \\
Silicon dioxide & $\mathrm{SiO}_{2}$ & $\mathbf{3 4 . 5 0}$ & $\mathbf{3 1 . 9 0}$ \\
Iron & $\mathrm{Fe}^{2+}$ & $\mathbf{3 . 3 6} \pm \mathbf{0 . 0 6}$ & $\mathbf{4 . 5 6} \pm \mathbf{0 . 0 6}$ \\
Zinc & $\mathrm{Zn}^{2+}$ & $\mathbf{0 . 0 0 7} \pm \mathbf{0 . 0 0 1}$ & $\mathbf{0 . 0 7 7} \pm \mathbf{0 . 0 0 1}$ \\
Manganese & $\mathrm{Mn}^{2+}$ & $\mathbf{0 . 0 4 7} \pm \mathbf{0 . 7}$ & $\mathbf{0 . 0 1 6} \pm \mathbf{0 . 0 8}$ \\
Copper & $\mathrm{Cu}^{2+}$ & $<1$ & $<1$ \\
Selenium & $\mathrm{Se}^{2-}$ & $\mathbf{0 . 0 5} \pm \mathbf{0 . 0 1}$ & $\mathbf{0 . 0 3} \pm \mathbf{0 . 0 1}$ \\
Osmolality $[\mathrm{mOsm} / \mathrm{kg}]$ & & $\mathbf{2 5 8 . 6 6} \pm \mathbf{6 . 3 3}$ & $\mathbf{5 7 . 3 3} \pm \mathbf{0 . 6 6}$ \\
\hline
\end{tabular}

Values are expressed in $\mathrm{mg} / \mathrm{L}$ except $\mathrm{SiO}_{2}$ in $\mathrm{mg} / \mathrm{kg}$. ND: not detected.

\subsection{Preparation of TW liposomal formulations}

\subsubsection{Conventional liposomes preparation}

Conventional liposomes loaded with thermal waters (TW-Lip) were prepared by the combined method of thin lipid film hydration with sonication. Liposomes were formulated with PC:PG:CH in 80:20:50 molar ratio, respectively (Ciobanu, Heurtault et al. 2007). Briefly, the desired amounts from each lipid stock solution were introduced into a Pyrex tube and organic solvents were removed on rotary evaporator (Bûchi, Switzerland) under reduced pressure, room temperature and moderate speed for $1 \mathrm{~h}$. The obtained dried thin lipid film was suspended in thermal water, BTW and STW separately, at a final lipid concentration of $15 \mathrm{mM}$ and agitated on a Vortex mixer for $10 \mathrm{~min}$. The obtained suspension of multilamellar liposomes (MLV) was further sonicated to obtain small unilamellar vesicles (SUV). A Vibra Cell 75041 ultrasonicator (750 W, $20 \mathrm{kHz}$, Fisher Bioblock Scientific, Illkirch, France) equipped with a $3 \mathrm{~mm}$-diameter tip probe set at $40 \%$ amplitude (sonication strength) was used with a sequence of $1 \mathrm{~s}$ of sonication and $2 \mathrm{~s}$ rest for 50 min under a continuous flow of argon. The suspension was kept cooled in a water bath to avoid heat generated during sonication. The SUV liposomes were then centrifuged at $13000 \mathrm{~g}$ to remove titane residues. The resulting samples (BW-Lip and SW-Lip) were stored at $4{ }^{\circ} \mathrm{C}$ until use. Control liposomes were formulated with the same method and the same lipid 
composition hydrated in $10 \mathrm{mM}$ Hepes buffer ( $\mathrm{pH} 7.4)$ containing $5 \%$ (w/v) sorbitol. The composition and molar ratio of conventional liposome vesicles are shown in Table 2.

\subsubsection{Deformable liposomes preparation}

TW loaded deformable liposomes (TW-DLip) were prepared by extrusion through polycarbonate filters as described before by Kakhi and al (Kakhi, Frisch et al. 2016) with slight modifications. Briefly, $15 \mathrm{mM}$ of PC stock solution was evaporated under vacuum in a Pyrex tube for $1 \mathrm{~h}$. The dry lipid film was then dispersed in ethanol (8.5\% ethanol volume/final volume) by mechanical shaking for $2 \mathrm{~min}$. Thermal water containing $5.4 \mathrm{mM}$ SDC was then added to the suspension in order to obtain a final concentration of $2.7 \mathrm{mM}$ of the surfactant. The suspension was agitated for 10 min to hydrate the lipid film, which resulted in the formation of MLVs. These MLVs were manually extruded through a $100 \mathrm{~nm}$ pore size polycarbonate membrane, at room temperature (above $25^{\circ} \mathrm{C}, \mathrm{T}_{\mathrm{m}}$ of PC), for 20 cycles to produce SUVs using an extrusion device (LiposoFastBasic, Avestin, Canada). The resulting suspensions were then stored at $4{ }^{\circ} \mathrm{C}$ until use.

Deformable liposome controls were formulated with the same method and the same membrane composition hydrated in 10mM Hepes buffer ( $\mathrm{pH} 7.4$ ) containing $5 \%(\mathrm{w} / \mathrm{v})$ sorbitol. The composition and molar ratio of deformable liposomes is shown in Table 2.

\section{Table 2}

Denomination and composition of liposome formulations.

\begin{tabular}{lllllll}
\hline Denomination & \multicolumn{5}{c}{ Molar ratio } & \multirow{2}{*}{ Hydration vehicle } \\
\cline { 2 - 5 } & \multicolumn{5}{c}{ Lipids } & \multicolumn{3}{c}{ Edge activator } & \\
\cline { 2 - 5 } & PC & PG & CH & SDC & STW \\
SW-Lip & $\mathbf{8 0}$ & 20 & 50 & - & STW; 7 \% v/v ethanol \\
SW-DLip & $\mathbf{8 3}$ & - & - & 27 & \\
\hline BW-Lip & $\mathbf{8 0}$ & 20 & $\mathbf{5 0}$ & - & BTW \\
BW-DLip & $\mathbf{8 3}$ & - & - & 27 & BTW; 7 \% v/v ethanol \\
\hline Hepes-Lip & $\mathbf{8 0}$ & 20 & $\mathbf{5 0}$ & - & Hepes \\
Hepes-DLip & $\mathbf{8 3}$ & - & - & 27 & Hepes; $7 \%$ v/v ethanol
\end{tabular}

PC: phosphatidylcholine, PG: phosphatidylglycerol, CH: cholesterol, SDC: sodium deoxycholate, , STW: Salhine thermal water, BTW: Baraka thermal water, Lip: liposomes, DLip: deformable liposomes.

\subsection{Physicochemical characterization of TWs loaded liposome formulations}

\subsubsection{Particle size}

The hydrodynamic diameter and the polydispersity index (PdI) of the different liposome formulations were measured in triplicate at $25^{\circ} \mathrm{C}$ by dynamic light scattering (DLS) using a Zetasizer Nano-ZS (Malvern instruments, Orsay, France) with a scattering angle of $173^{\circ}$. Samples were diluted in the same encapsulated TW at a 1/100 volume ratio and were placed in 
disposable plastic micro-cuvettes (Brand $\mathrm{GmbH}$, Germany). The represented data are the average of at least 3 measurements done \pm standard deviation (standard error) (SD).

\subsubsection{Zeta Potential}

The zeta potential of TWs-loaded liposome formulations was measured at $25^{\circ} \mathrm{C}$ using Zetasizer Nano-ZS (Malvern instruments, Orsay, France). Samples were diluted at 1/20 volume ratio in purified $18 \mathrm{M} \Omega$ ultrapure-water. Measurements were performed in triplicate to determine the zeta potential average values \pm SD.

\subsubsection{Physical stability}

Formulated suspensions of BTW- and STW-loaded conventional and deformable liposomes were stored at $4{ }^{\circ} \mathrm{C}$ during 30 days. Their macroscopic behavior was visually inspected. The particle size and PdI values were checked at $10^{\text {th }}, 20^{\text {th }}$ and $30^{\text {th }}$ day of storage. Mean values were used for the analysis of the data.

\subsection{Encapsulation efficiency}

The efficiency of encapsulation of TWs into liposome formulations (EE\%) was assessed by determining the concentration of free minerals contained in the non-encapsulated external TWs by dialysis. $1 \mathrm{~mL}$ of each liposome formulation $(15 \mathrm{mM})$ was dialyzed using a cellulose membrane with an Mw cutoff of 12-14 $000 \mathrm{kD}$ (Spectra/Pore®, Spectrum Laboratories Inc. USA) against $250 \mathrm{~mL}$ deionized water for $12 \mathrm{~h}$, under stirring at $4{ }^{\circ} \mathrm{C}$. Chloride was chosen as a trace element to determine the amount of non-encapsulated minerals (Ribeiro, Araújo et al. 2015). Its concentration was determined by Mohr method (Shukla and Arya 2018). Particles size compared before and after dialysis proved their stability. The following equation was used to calculate the $\mathrm{Cl}^{-}$encapsulation efficiency that represents also the efficiency encapsulation of TWs into formulated liposome suspensions:

$$
E E \%=\frac{\text { Concentration of entrapped } \mathrm{Cl}^{-}}{\text {Concentration of } \mathrm{Cl}^{-} \text {in } \mathrm{TW}} \times 100
$$

\subsection{Cell culture}

RAW 264.7 mouse macrophage cells were obtained from ATCC® TIB-71TM. Cells were cultured in DMEM supplemented with stabilized glutamine, $5 \%$ (v/v) FBS containing 1000 $\mathrm{U} / \mathrm{mL}$ of penicillin and $100 \mu \mathrm{g} / \mathrm{mL}$ streptomycin at $37{ }^{\circ} \mathrm{C}$ under a $5 \% \mathrm{CO}_{2} 95 \%$ humidified air atmosphere. Cells were harvested at around $90 \%$ confluence using trypsin $(0.5 \mathrm{~g} / \mathrm{L})$-EDTA $(0.2$ $\mathrm{g} / \mathrm{L})$ for $5 \mathrm{~min}$ at $37{ }^{\circ} \mathrm{C}$ and re-suspended in culture medium to the desired dilution. For all experiments, cells were seeded in a 96 -well plate at $5 \times 10^{4}$ cells/well.

\subsection{In-vitro inflammatory investigation of TWs and TWs-liposome formulations}

For the pro-inflammatory experiments, the cells were diluted and dispensed to 96-well plated, 50 $\mu \mathrm{L} /$ well $\left(5 \times 10^{4}\right.$ cells/well $)$. The cells were allowed to adhere for $24 \mathrm{~h}$ at $37^{\circ} \mathrm{C}$ with $5 \% \mathrm{CO}_{2}$ and 
the non-adherent cells were washed away. Fresh medium was added to the cells, and the cells were treated with liposome encapsulated (final concentration 3.125 to $100 \mu \mathrm{M}$ for conventional and deformable liposomes) or free BTW and STW (final volume 1 to $50 \mu \mathrm{L} / w e l l$ ). The effects of lipopolysaccharide (LPS, $50 \mathrm{ng} / \mathrm{mL}$ ) and DMSO (5 and $10 \%$ ) were also tested as controls. After an overnight incubation at $37{ }^{\circ} \mathrm{C}$, cell supernatants were used to measure pro-inflammatory compound (nitric oxide (NO)) and inflammation-related cytokine (TNF- $\alpha$ ). The cell viability was evaluated with microscopy visualisation.

To evaluate the anti-inflammatory activity after the same first steps until washing the cells, DMEM containing two showed volume of BTW and STW (10 and 50 $\mu$ L) and serial concentrations of BTW and STW loaded conventional and deformable liposomes (10, 50 and 100 $\mu \mathrm{M}$ respectively) was added (final volume $100 \mu \mathrm{L} /$ well). After an incubation of 1,2 and $4 \mathrm{~h}$ at $37{ }^{\circ} \mathrm{C}$, inflammatory response was stimulated by adding $50 \mathrm{ng} / \mathrm{mL}$ LPS for $24 \mathrm{~h}$ at $37{ }^{\circ} \mathrm{C}$. The negative and positive controls were the cells incubated in culture media non induced and induced with LPS, respectively. The cell supernatants were collected to determine nitrite oxide and TNF$\alpha$ production.

\subsection{Determination of nitrite concentration}

The concentration of nitrite $\left(\mathrm{NO}_{2}{ }^{-}\right)$, a stabilized form of nitric oxide (NO), was measured using Griess method (Hardy, Seguin et al. 2018). Briefly, $40 \mu \mathrm{L}$ of culture supernatants from each well were mixed with $60 \mu \mathrm{L}$ of Griess reagent (equal volumes v/v of $58.1 \mathrm{mM}$ P-aminobenzenesulfonamide in $30 \%$ acetic acid and $3.9 \mathrm{mM} \mathrm{N}$-(1-naphtyl) ethylenediamine dihydrochloride in $60 \%$ acetic acid) in a separate 96-well plate. The absorbance of the colored product was measured at $\lambda=543 \mathrm{~nm}$, using a microliter plate photometer (Safas SP2000, Xenius 5801, Monaco). The method was standardized with a sodium nitrite curve ranging from 3.125 to 100 $\mu \mathrm{M}$.

\subsection{Estimation of TNF- $\alpha$ production}

TNF- $\alpha$ concentration in culture supernatants was evaluated by ELISA using commercially available reagents and following the manufacturer instructions. Purified anti-mouse TNF $\alpha$ antibody clone 1F3F3D4 and biotinylated anti-mouse TNF $\alpha$ antibody clone XT3/XT22 were purchased from eBiosciences/thermoFischer Scientific (Waltham, MA, USA). Avidin HRP was from Jackson (West Grove, PA, USA). Briefly, capture antibody was diluted to $1 \mu \mathrm{g} / \mathrm{mL}$ in a 0.05 $\mathrm{M} \mathrm{pH} 9.6$ carbonate/bicarbonate buffer and coated 1 night at $4^{\circ} \mathrm{C}$ before blocking with PBS $0.05 \%$ Tween $201 \%$ BSA $\left(1 \mathrm{~h}, 37^{\circ} \mathrm{C}\right)$. Samples were then diluted with PBS (1:20 and 1:5 for treated and untreated cells with LPS, respectively) and incubated with capture antibody (2h, $37^{\circ} \mathrm{C}$ ) before addition of the biotinylated detection antibody diluted to $0.5 \mu \mathrm{g} / \mathrm{mL}$ in PBS $0.05 \%$ Tween $201 \%$ BSA $\left(1 \mathrm{~h}, 37^{\circ} \mathrm{C}\right)$. Avidin HRP was then added $\left(45 \mathrm{~min}, 37^{\circ} \mathrm{C}\right)$ and revelation was conducted by addition of $1.25 \mathrm{mM}$ tetramethylbenzidine and $13.05 \mathrm{mM} \mathrm{H}_{2} \mathrm{O}_{2}$ in $0.1 \mathrm{M} \mathrm{pH}$ citrate buffer. Revelation was finally stopped by addition of $1 \mathrm{M} \mathrm{HCl}$ and absorbance was 
measured at $450 \mathrm{~nm}$ and compared to a calibration curve constructed from standard TNF- $\alpha$ solutions.

\section{Results and discussion \\ 3.1.Characterization of thermal waters}

Both BTW and STW can be classified into neutral highly mineralized thermal waters, according to their neutral $\mathrm{pH}$, relatively high conductivities and hot temperatures (Table 1) (Ghersetich and Lotti 1996, Nasermoaddeli and Kagamimori 2005). BTW belongs to the sodium-sulfate type $\left(\left[\mathrm{Na}^{+}\right]=461.79 \mathrm{mg} / \mathrm{L},\left[\mathrm{SO}_{4}^{2-}\right]=1742.92 \mathrm{mg} / \mathrm{L}\right)$ while $\mathrm{STW}$ belongs to the sodium-chloride $\left(\left[\mathrm{Cl}^{-}\right]=4190 \mathrm{mg} / \mathrm{L},\left[\mathrm{Na}^{+}\right]=2943 \mathrm{mg} / \mathrm{L}\right)$ and calcium-sulfate $\quad$ types $\quad\left(\left[\mathrm{SO}_{4}^{2-}\right]=\right.$ $\left.1220 \mathrm{mg} / \mathrm{L},\left[\mathrm{Ca}^{2+}\right]=280 \mathrm{mg} / \mathrm{L}\right)$. Moreover, both BTW and STW are highly enriched in magnesium, potassium and bicarbonate ions. They show an important mineralization in minor trace elements particularly represented by strontium, iron, selenium and manganese. Related to their osmolality values (Table 1), BTW and STW can be classified as hypotonic and isotonic water, respectively. These mineral compositions reflect the possible physiological mechanism of BTW and STW in contact with skin. Several reports have previously demonstrated the relation between rich content of TWs in major and minor minerals and their physiological activities as immune modulatory, antioxidant and anti-inflammatory activities (Celerier, Litoux et al. 1995, Joly, Galoppin et al. 2000, Verdy, Branka et al. 2012). In view of these results, BTW and STW can be considered as potential products for the treatment of skin pathologies, within the framework of dermo-cosmetics.

\subsection{Physicochemical characteristics of TW liposome suspensions}

Conventional liposomes composed of PC:PG:CH (80:20:50 molar ratio) and encapsulating BTW and STW were produced by lipid film-hydration method, followed by sonication. Both BW-Lip and SW-Lip showed a narrow particle size distribution with a mean diameter around $60 \mathrm{~nm} \pm 12$ $\mathrm{nm}$ and a PdI $\leq 0.2$ (Table 3). It was previously reported that the presence of $\mathrm{CH}$ in liposomes composition achieve to small molecular geometry and hydrophobicity desirable to form closed small bilayer vesicles (Manosroi, Wongtrakul et al. 2003). In addition, sonication reduced the size of liposomes to the nanorange and allowed to the formation of uniform size (Yamaguchi, Nomura et al. 2009).

Deformable liposomes encapsulating BTW and STW were formulated with PC:SDC (73:27 molar ratio) in presence of ethanol by lipid film-hydration method followed by extrusion. Deformable liposomes (BW-DLip and SW-DLip) showed a mean diameter size of $134 \pm 15 \mathrm{~nm}$ (Table 3), closed to the pore size of the membranes used for the extrusion. The size of deformable liposomes was greater than conventional liposomes. Deformable liposome suspensions were mono dispersed with a $\mathrm{PdI} \leq 0.07$. It is well known that extrusion method produces unilamellar liposome suspensions of spectacularly homogeneous size in the nano-range of 30-200 nm (Alves, Cusick et al. 2013). 
Zeta potential is an important indicator of liposomes surface charge that allows predicting about physical stability. Conventional liposomes showed anionic charges of $-30.23 \pm 8.61 \mathrm{mV}$ and $23.00 \pm 7.12 \mathrm{mV}$ for SW-Lip and BW-Lip (Table 3), respectively, related to the negative charge of PG present in the formulations. Deformable liposomes exhibited a negative zeta potential ranged between $-8.28 \pm 8 \mathrm{mV}$ and $-11.87 \pm 7 \mathrm{mV}$ (Table 2), related to the presence of an anionic surfactants (SDC). Compared to the zeta potential of conventional and deformable liposomes prepared in Hepes medium (around $-80 \mathrm{mV}$ and $-30 \mathrm{mV}$, respectively, data not shown), liposomes formulated in TWs exhibit a less negatively zeta potential, which can be due to the interaction of metal ions present in the TWs with charged phospholipids via Coulombic interactions (Le, Houri et al. 2019). According to literature, the net negative surface charges of membranes of anionic lipids increases cations concentration near the lipid-water interface according to the Gouy-Chapman theory of the electrical double layer (McLAUGHLIN, Mulrine et al. 1981). The counterions do not penetrate the headgroup zone but form a layer over the membrane instead (Le, Houri et al. 2019). As a result, the electric double layer will undergo such a high compression that the zeta potential tends towards zero. The electrostatic shielding of liposome charged surfaces can suppress the electrostatic repulsion and the electrophoretic mobility of particles. In this case, flocculation of formulated liposomes is suspected.

\section{Table 3}

Physicochemical characteristics (vesicle diameter, PdI, zeta potential) and encapsulation efficiency of liposome formulations $(n=3)$.

\begin{tabular}{lllll}
\hline Formulation & $\begin{array}{l}\text { Vesicle diameter } \\
(\mathrm{nm}) \pm \mathrm{SD}\end{array}$ & PdI \pm SD & $\begin{array}{l}\text { Zeta potential } \\
(\mathrm{mV}) \pm \mathrm{SD}\end{array}$ & EE \% \pm SD \\
\hline SW-Lip & $\mathbf{6 6 . 0 0} \pm \mathbf{8 . 0 0}$ & $\mathbf{0 . 1 5 0} \pm \mathbf{0 . 0 1}$ & $\mathbf{- 3 0 . 2 3} \pm \mathbf{8 . 6 1}$ & $\mathbf{9 6 . 5 1} \pm \mathbf{1 . 1 4}$ \\
SW-DLip & $\mathbf{1 3 4 . 1 0} \pm \mathbf{1 5 . 0 0}$ & $\mathbf{0 . 0 7 0} \pm \mathbf{0 . 0 4}$ & $-\mathbf{1 1 . 8 7} \pm \mathbf{7 . 0 3}$ & $\mathbf{8 5 . 2 0} \pm \mathbf{1 . 1 0}$ \\
\hline BW-Lip & $\mathbf{6 0 . 0 0} \pm \mathbf{9 . 0 0}$ & $\mathbf{0 . 1 8 1} \pm \mathbf{0 . 0 1}$ & $\mathbf{- 2 3 . 0 0} \pm \mathbf{7 . 1 2}$ & $\mathbf{9 5 . 0 2} \pm \mathbf{1 . 8 9}$ \\
BW-DLip & $\mathbf{1 3 4 . 0 0} \pm \mathbf{1 3 . 0 0}$ & $\mathbf{0 . 0 2 7} \pm \mathbf{0 . 0 1}$ & $\mathbf{- 8 . 2 8} \pm \mathbf{8 . 0 3}$ & $\mathbf{7 2 . 5 2} \pm \mathbf{2 . 2 2}$ \\
\hline
\end{tabular}

\subsection{Physical stability}

Liposome dispersions are mainly stabilized against sedimentation by electrostatic repulsion (Heurtault, Saulnier et al. 2003). In our case, the strong electrolyte charge in BTW and STW considerably reduces the electrostatic repulsion between the liposomes, which negatively influences their stability by flocculation mechanism (Heurtault, Saulnier et al. 2003). However, the absence of electrostatic repulsion between liposomes will not necessarily result in aggregate formation or fusion of the colloidal particles, regardless the hydration energies (Crommelin 1984). Visual inspection of the prepared liposome dispersions stored at $4^{\circ}$ during 30 days did not reveal any change in the appearance of conventional liposome dispersions. However, precipitation appeared in deformable liposome suspensions after $48 \mathrm{~h}$ of storage. In the case of 
conventional liposome suspensions, flocculation probably generated the formation of a threedimensional network holding the entire volume of the system, which might be responsible of the suspensions stability against sedimentation. In the case of deformable liposomes, the formation of the three-dimensional network is unlikely which may be attributed to the deformability of the liposome membrane.

Table 4 shows that the average vesicles size mainly of SW-Lip slightly increased during the first 10 days of storage and almost stabilized over the subsequent days, which can be induced by the peroxidation of lipids (Heurtault, Saulnier et al. 2003). Other author have observed the same size increase of liposomes prepared with deionized water (Wang, Swing et al. 2020). Stability of liposomes size indicates the stability of encapsulated TWs volume. Manosroi et al. (Manosroi, Wongtrakul et al. 2003) reported that $\mathrm{CH}$ is an important constituent of liposomes for the stabilization of the vesicular bilayer membrane and to achieve suitable hydrophobicity. Basically, the stability of ionic strength inside and outside the liposomes maintains the stability of liposomes with high retention rates (Wang, Swing et al. 2020). The PdI values of all formulations were $<0.3$ indicating monodispersity with a narrow size distribution and perfect suspension homogeneity.

\section{Table 4}

Evolution of the average particle diameter and the PdI of TWs-loaded conventional and deformable liposomes stored at $4{ }^{\circ} \mathrm{C}$ as a function of time. Data are mean value \pm standard deviation $(n=3)$.

\begin{tabular}{lllllllll}
\hline Formulation & \multicolumn{2}{l}{ Particle diameter $(\mathrm{nm})$} & & & & \\
& \multicolumn{2}{l}{ PdI } & & \\
& Day 1 & Day 10 & Day20 & Day30 & Day1 & Day10 & Day20 & Day30 \\
\hline BW-Lip & $\mathbf{5 9} \pm \mathbf{8}$ & $\mathbf{6 4} \pm \mathbf{8}$ & $\mathbf{6 7} \pm 7$ & $\mathbf{6 5} \pm 7$ & $\mathbf{0 . 1 8 1}$ & $\mathbf{0 . 2 1 6}$ & $\mathbf{0 . 1 8 2}$ & $\mathbf{0 . 1 8 3}$ \\
SW-Lip & $\mathbf{6 6} \pm \mathbf{8}$ & $\mathbf{8 3} \pm 9$ & $\mathbf{8 2} \pm 9$ & $\mathbf{8 1} \pm \mathbf{8}$ & $\mathbf{0 . 1 5 0}$ & $\mathbf{0 . 1 5 8}$ & $\mathbf{0 . 1 7 8}$ & $\mathbf{0 . 1 7 0}$ \\
\hline BW-DLip & $\mathbf{1 3 6} \pm \mathbf{8}$ & $\mathbf{1 4 0} \pm \mathbf{3}$ & $\mathbf{1 4 0} \pm \mathbf{2}$ & $\mathbf{1 4 5} \pm \mathbf{1 1}$ & $\mathbf{0 . 0 7 4}$ & $\mathbf{0 . 0 8 8}$ & $\mathbf{0 . 0 9 0}$ & $\mathbf{0 . 1 0 4}$ \\
SW-DLip & $\mathbf{1 3 6} \pm \mathbf{3}$ & $\mathbf{1 4 0} \pm \mathbf{2}$ & $\mathbf{1 4 1} \pm \mathbf{1}$ & $\mathbf{1 4 2} \pm \mathbf{8}$ & $\mathbf{0 . 0 4 4}$ & $\mathbf{0 . 0 2 3}$ & $\mathbf{0 . 0 7 1}$ & $\mathbf{0 . 0 8 4}$ \\
\hline
\end{tabular}

\subsection{Encapsulation efficiency}

BTW and STW encapsulation rates exceeded $90 \%$ in conventional liposomes while ranged between 73 and $85 \%$ in the case of deformable liposomes (Table 3). The observed results presented comparatively higher EE\% in conventional than deformable liposomes. It may be justified on the basis of the presence of $\mathrm{CH}$ in the composition of conventional liposomes, which yielded higher EE\% of hydrophilic substances by decreasing membrane permeability (Kulkarni, Betageri et al. 1995). Moreover, the lower EE\% of deformable liposomes might be related to membrane fluidity and permeability induced by the intercalation of ethanol into the PC polar head group environment (Fan, Li et al. 2013). The formulation method and the vesicles size might also have influenced the encapsulated volume of TWs. 
For further in-vitro studies, the encapsulated volume of BTW and STW in liposome dispersions diluted at 10, 50 and $100 \mu \mathrm{M}$ was calculated using the EE \% values (Table 5).

\section{Table 5}

BTW and STW volumes encapsulated in $1.5 \mathrm{~mL}$ of 10, 50, 100 and $15000 \mu \mathrm{M}$ of liposomes (concentrations expressed in phospholipids)

\begin{tabular}{|l|c|c|c|c|c|}
\cline { 3 - 6 } \multicolumn{2}{c|}{} & \multicolumn{4}{c|}{$\begin{array}{c}\text { Estimated TWs volume encapsulated in different } \\
\text { concentrations of liposomes }(\mu \mathrm{L})\end{array}$} \\
\hline Liposomes & EE \% & $15 \mathrm{mM}$ & $100 \mu \mathrm{M}$ & $50 \mu \mathrm{M}$ & $10 \mu \mathrm{M}$ \\
\hline SW-Lip & 95.90 & 1438.50 & 9.59 & 4.80 & 0.96 \\
\hline SW-DLip & 85.20 & 1169.37 & 7.80 & 3.90 & 0.78 \\
\hline BW-Lip & 93.66 & 1404.90 & 9.37 & 4.68 & 0.94 \\
\hline BW-DLip & 73.90 & 1014.28 & 6.76 & 3.38 & 0.68 \\
\hline
\end{tabular}

\subsection{Effect of TWs and TWs-loaded liposome formulations on NO and TNF-a production by RAW 264.7 macrophages}

To evaluate the pro-inflammatory effect of BTW and STW, free and loaded liposome formulations, a model analyzing the activation of RAW 264.7 cells was used. Figure 1 (A, B and C) shows the NO production in RAW 264.7 cells incubated with increased volumes of free BTW and STW, and increased concentrations of BTW and STW loaded conventional and deformable liposomes. We can observe that whatever the added volume of BTW and STW and concentration of liposomes charged with BTW and STW, the cells produced the same level of NO as the untreated cells $(1.03 \pm 0.89 \mu \mathrm{M})$. In contrast, the cells treated with LPS, used as reference of inflammation, produced a high concentration of NO $(17.04 \pm 2.66 \mu \mathrm{M})$. These results indicated that both free and encapsulated BTW and STW did not induced the NO production.

The effect of free and encapsulated BTW and STW on the TNF- $\alpha$ secretion at the used volumes and concentrations is shown in Figure 1 (D, E and F). Low concentrations of TNF- $\alpha$ were produced by cells treated with the used volumes and concentration of free and encapsulated BTW and STW, respectively. These concentrations were approximately the same as the TNF- $\alpha$ concentration produced by untreated cells $(84.74 \pm 23.69 \mathrm{pg} / \mathrm{mL})$. Whereas in a case of cell inflammation by LPS, the concentration of TNF- $\alpha$ produced is 86 times greater $(7242.63 \pm$ $641.00 \mathrm{pg} / \mathrm{mL}$ ). These results clearly demonstrate that the used volumes and concentrations of free and encapsulated BTW and STW did not induced the TNF- $\alpha$ secretion in RAW 264.7 cells.

The viability of the macrophages after treatment with free and liposomal BTW and STW was visualized under microscopy and compared with the viability of untreated cells and cells treated with DMSO (5\% and $10 \%$ ). The microscopic visualization showed no difference in morphology between untreated and RAW cells cultured with different concentrations of TWs and TWLiposome formulations (data not shown). 
These results suggest that free and encapsulated BTW and STW have no pro-inflammatory effect and volumes of 10 and $50 \mu \mathrm{L}$ (BTW and STW) and concentrations of 10, 50 and $100 \mu \mathrm{M}$ of conventional and deformable liposomes charged with BTW and STW were chosen for subsequent experiments.

\subsection{Effects of TWs, TWs-Lip and TWs-DLip on LPS-activated RAW 264.7 macrophage cell production of $\mathrm{NO}$ and $\mathrm{TNF} \alpha$}

As thermal waters have shown their positive effect on skin diseases caused by inflammation (Largeau 1881, Boughlali 2003, Ali 2008), the potential effect of TWs, free and encapsulated in various formulations on macrophages activation was evaluated. For this, macrophages were treated for $1 \mathrm{~h}, 2 \mathrm{~h}$ and $4 \mathrm{~h}$ with $10 \%$ and $50 \%$ of Baraka and Salhine thermal waters (BTW and STW) compared to Hepes medium, and then activated by LPS (50 ng/mL) for $24 \mathrm{~h}$. Then conventional and deformable liposomes charged with both TWs were prepared (BW-Lip, SWLip, BW-DLip and SW-DLip). The potential of encapsulated TWs in various formulations was also tested on macrophages activation with the same protocole. LPS-induced untreated cells were taken as negative control and reference sample in all the experiments.

\subsubsection{Effect of BTW and STW on NO production and TNF- $\alpha$ expression in LPS- induced macrophages}

As shown in Figure 2A, LPS clearly induced considerable NO production in untreated RAW 264.7 cells $(15.34+/-2.08 \mu \mathrm{M})$. As expected, the pretreatment of cells with BTW and STW shows a clear reduction in NO production in a dose-dependent manner. Interestingly BTW showed a more pronounced inhibitory effect than STW, which might be justified by the difference in the mineral content. Taking as an example, the pretreatment of cells with $10 \mu \mathrm{L}$ and $50 \mu \mathrm{L}$ of BTW for $4 \mathrm{~h}$ before LPS addition, reduced the NO production by $37 \%$ and $75 \%$, respectively, when LPS was added after $4 \mathrm{~h}$ of TWs addition. On the other hand, NO levels decreased but remained unchanged during incubations with Hepes medium and do not exceeded a reduction by $24 \%$. In addition, the pre-incubation durations had practically no impact on the inhibitory effect of BTW and STW. Thus, a pretreatment duration of $1 \mathrm{~h}$ was sufficient for BTW and STW to attenuate the inflammatory effect of LPS. It has been previously revealed that a deep-sea water containing enhanced $\mathrm{Mg}^{2+}$ and $\mathrm{Ca}^{2+}$ concentrations strengthened the inhibitory effect of chitosan oligosaccharides on iNOS expression in LPS-induced macrophages (Maxwell, Lee et al. 2017). Moreover, an in vitro study using human dermal fibroblasts and human skin explants showed that a rich sulphate carbonate-calcium magnesium thermal water prevented the ROS induced damage (Joly, Branka et al. 2014). Numerous studies have also evidenced the effect of TWs on the activities of enzymes involved in the skin's defense system against free radicals inflammatory response (Richard, Guiraud et al. 1990, Cadi, Beani et al. 1991, Bruneau, Bernard et al. 1996). In accordance to these literatures, it can be speculated that the rich composition of BTW and STW with previous mentioned chemical elements might modulate the NO production in LPS induced macrophages. 
We next investigated the effects of BTW and STW on the expression of TNF- $\alpha$ in LPS-induced RAW 264.7 cells. The expression of TNF- $\alpha$ cytokine was easily detected in RAW cell supernatants by ELISA. Treatment with both BTW and STW attenuated the LPS-induced increase in TNF- $\alpha$ secretion in a concentration-dependent manner (Fig. 2B). The inhibitory effect of TWs was the same independently of pre-incubation times. It can be also noticed that BTW at $50 \mu \mathrm{L}$ seems to prevent more effectively the release of TNF- $\alpha$ than STW at least until $4 \mathrm{~h}$. This observed difference might be attributed to the rich composition of BTW with zinc $(0.077 \pm 0.001$ $\mathrm{mg} / \mathrm{L}$ ) (Table 2). The anti-inflammatory effect of zinc was previously described (Prasad 2014). In addition, BTW and STW have a common composition with trace elements, sodium bicarbonate, and boron ingredients which might be responsible for the suppression of pro-inflammatory cytokines. Selenium and strontium, which are highly present in STW, proved an inhibitory effect on IL-1, IL-6 and TNF- $\alpha$ inflammatory cytokine production by keratinocytes in atopic dermatitis lesions (Celerier, Litoux et al. 1995). Moreover, La Roche-Posay, selenium-rich water, has significantly inhibited TNF- $\alpha$ and IL-1 production in Langerhans cells (LCs) (Wollenberg, Richard et al. 1992), related to the action of containing minerals on the functional mutation of LCs. Other common chemical components in BTW and STW were sodium, chloride and sulphate. Application and balneotherapy with sodium-chloride sulphate mineral waters were found to have potential anti-inflammatory effects on human skin during induced inflammatory reactions and psoriatic plaques (Hercogova, Stanghellini et al. 2002, Tsoureli-Nikita, Menchini et al. 2002). Furthermore, researchers have also demonstrated the effectiveness of an hypotonic thermal water to significantly down regulated the intracellular levels of TNF- $\alpha$ in cultured human psoriatic keratinocytes (Dal Pra, Chiarini et al. 2007). Moreover, a study conducted on human endothelial cells had noted a significant inhibition of the TNF- $\alpha$-induced E-selectin and ICAM-1 expression in the presence of thermal water (Castex-Rizzi, Charveron et al. 2011). As well, treatment with thermal water from Korea spring decreased the expression of pro-inflammatory cytokines in HaCaT cells under Toll-like receptor (TLR) (Lee, Choi et al. 2012). Overall, data obtained in the current study are in perfect agreement with previous findings which suggest that BTW and STW have anti-inflammatory effects.

\subsubsection{Effect of BTW and STW-loaded conventional liposomes on NO production and TNF- $\alpha$ expression in LPS-induced macrophages}

First, we investigated the effects of conventional liposome formulations on LPS-induced NO production. BW-Lip and SW-Lip inhibited considerably the NO release in a dose-dependent manner (Fig. 3A). The previous pro-inflammatory study ensures that the reduction in NO production was not due to cells damage. A high inhibitory effect of both BW-Lip and SW-Lip was most noticeable after $4 \mathrm{~h}$ of pre-incubation. The data showed also that BW-Lip and SW-Lip at 10 and $50 \mu \mathrm{M}$ have practically the same inhibitory effect, while at $100 \mu \mathrm{M}$, BW-Lip showed most noticeable the more efficient effect. The difference in the efficiency of BW-Lip and SW-Lip at $100 \mu \mathrm{M}$ might be explained by the more effective inhibitory effect of BTW, previously noted. 
We next tested the effect of BW-Lip and SW-Lip on the TNF- $\alpha$ production in LPS-induced RAW 264.7 cells compared to untreated cells (Fig. 3B). Firstly, the results showed that liposome formulations did not affect the expression of TNF- $\alpha$ by RAW 264.7 cells non-stimulated with LPS. Furthermore, BW-Lip and SW-Lip reduced effectively the level of TNF- $\alpha$ expressed in LPS-stimulated RAW 264.7 cells in concentration- and time-dependent manner. As a general trend, the expression of TNF- $\alpha$ was effectively inhibited as the TW-Lip concentration increased and the preincubation time decreased. Importantly, these experiments demonstrate that BW-Lip had a greater inhibitory effect than SW-Lip.

The most remarkable result was observed when comparing the effects of BW-Lip and SW-Lip at $100 \mu \mathrm{M}$ (Fig. 3A \& B), that encapsulate around $10 \mu \mathrm{L}$ of BTW and STW, respectively (Table 5), with the effects of $10 \mu \mathrm{L}$ non-encapsulated BTW and STW (10\%, v/v) (Fig. 2A \& B). Interestingly, TWs encapsulated in liposomes inhibited more efficiently the NO and TNF- $\alpha$ release than free TWs, which revealed that liposomes strength the anti-inflammatory effect of encapsulated TWs. One of the possible explanations for these results can be the mechanism of encapsulated minerals uptake. The cellular uptake of liposomes was found to be mediated by adsorption of liposomes onto the cytoplasmic membrane and subsequent endocytosis (Allen, Austin et al. 1991). This allows to introduce to macrophage cells, a large quantity of encapsulated drugs, that increases with increasing liposome concentration and incubation time at $37^{\circ} \mathrm{C}$ (Allen, Austin et al. 1991). In addition to the effect of the TWs compositions with minerals, the lipid composition of vesicles might also participate in the inhibition of TNF- $\alpha$ expression. An earlier study from Treede et al. showed that PC had a broad ability to block many processes linked to the pro-inflammatory response in human Caco- 2 cells treated with TNF- $\alpha$ (Treede, Braun et al. 2007). Further, the research group of Klein et al. showed that PG-loaded liposomes significantly decreased the TNF- $\alpha$ production in LPS-induced macrophages (Klein, Mauch et al. 2020). Moreover, the resulting catabolites of liposome membranes are exported from the lysosome and reused as raw materials in cellular metabolism (Kolter and Sandhoff 2010).

Our data show that the encapsulation of TWs in phospholipid vesicles strengthens their antiinflammatory effect, which could be attributed on the one hand by increasing their bioavailability and on the other hand by adding the anti-inflammatory effect of used phospholipids.

Our findings are in good agreement with previous results reported the antioxidant power of free and encapsulated TWs in a vesicular composition on human fibroblast cell cultures (Laugier, Ribier et al. 1993). It was found that glutathione peroxidase (GPX) and superoxide dismutase (SOD) enzymes, which are two links in the radical detoxification chain, have their activities potentiated in the presence of encapsulated TWs. As for us, authors have concluded that the encapsulation of TWs in lipid vesicles potentiated its effects, particularly its antiradical and antioxidant activities. Further tests carried out human keratinocytes had confirmed the moisturizing and the anti-inflammatory effects of SUV liposomes charged with a strontium rich thermal water (Nunes and Tamura 2012, Tamura, Pedroso et al. 2012). 


\subsubsection{Effect of BTW and STW-loaded deformable liposomes on NO production and TNF- $\alpha$ expression in LPS-induced macrophages}

In this part of experiments, we studied the effects of BTW- and STW-loaded deformable liposomes (BW-DLip and SW-DLip, respectively) on the NO and TNF- $\alpha$ cytokine production in LPS-activated RAW 264.7 cells with the same method previously described. Obtained results showed a reduced nitrite concentration in the presence of BW-DLip and SW-DLip in a concentration-dependent manner (Fig. 4A). However, a low decrease in NO production was observed in cells treated with $10 \mu \mathrm{M}$ of deformable liposomes, while the levels of NO produced by cells treated with $100 \mu \mathrm{M}$ of deformable liposomes are reduced to nearly the half of NO level produced by control cells. Besides, the NO production was strongly attenuated when the cells are incubated time with deformable liposomes for a short before the LPS-induction. The analysis of TNF- $\alpha$ production (Fig. 4B) has indicated that BW-DLip and SW-DLip inhibited dosedependently the release of TNF- $\alpha$. As already observed, deformable liposomes showed a stronger inhibitory effect on the TNF- $\alpha$ production at a short incubation time. In addition, no significant difference related to the encapsulated TWs was observed.

Compared to conventional liposomes, and taking in consideration the standard deviation values, deformable liposomes charged with TWs have practically the same inhibitory effect of NO and TNF- $\alpha$ production on LPS-induced RAW 264.7 cells as conventional liposomes charged with the same TWs. It must be also noted that even in absence of PG and $\mathrm{CH}$ from the lipid composition, deformable liposomes exert a marked inhibitory effect. This can confirm that registered antiinflammatory effects are dominated by the anti-inflammatory effects of minerals containing in encapsulated TWs.

As confirmed in previous in vitro studies, deformable liposomes could play significant roles in improving the effects of encapsulated drugs, such as anti-inflammatory (Barone, Cristiano et al. 2020) and antioxidant effects (Liu, Hu et al. 2013). Previous study revealed the efficiency of niosomes, composed of surfactants and $\mathrm{CH}$, entrapped with mineral water to enhance the skin absorption of minerals and the moisturizing effect of the encapsulated mineral water (Manosroi, Witkittilak et al. 2010).

\section{Conclusion}

In summary, we have successfully incorporated highly mineralized thermal waters into conventional and deformable liposomes using respectively sonication and extrusion techniques. The obtained results confirmed that both TWs-conventional and deformable liposomes have high encapsulation efficiency, nano-size and negative charge. The present study suggests that the high ionic strength of external environment mainly neutralized the zeta potential of the vesicles and affect the suspensions stability. During 30 days of storage, the conventional liposomes showed excellent stability, but sedimentation formation was observed in deformable liposomes. 
Moreover, all liposomal formulations were biocompatible and non-toxic for the RAW 264.7 cells. Additionally, the anti-inflammatory potential of the TWs-conventional and deformable liposomes in LPS-induced RAW 264.7 macrophages was studied and compared to those of free TWs. The current study provides clear evidence that TWs-entrapped conventional and deformable liposomes exert anti-inflammatory effects by preventing LPS-induced NO and TNF- $\alpha$ release from RAW 264.7 macrophages. All liposomal formulations exerted concentrationdependent anti-inflammatory activity, superior to activities of free TWs. We confirmed that incorporation of TWs in conventional and deformable liposomes enhances their biological potentials. Our results suggest that the TWs-entrapped phospholipid vesicles can be useful in the treatment of various inflammatory skin diseases.

\section{Acknowledgements}

The authors thank the Plateforme d'Analyse des Inorganiques (PAI), UMR 7178 CNRSUniversité de Strasbourg for analytical analysis of thermal waters. The authors thank the FrenchAlgerian scholarship program, PROFAS B, for its financial support. This work of the Interdisciplinary Institute HiFunMat, as part of the ITI 2021-2028 program of the University of Strasbourg, CNRS and Inserm, was supported by IdEx Unistra (ANR-10-IDEX-0002) and SFRI (STRAT'US project, ANR-20-SFRI-0012) under the framework of the French Investments for the Future Program.

\section{References}

Ali, T. (2008). "LES THERMES ET LE THERMALISME EN ALGERIE." La Revue des Sciences Commerciales 7(2): 11-26.

Allen, T., G. Austin, A. Chonn, L. Lin and K. Lee (1991). "Uptake of liposomes by cultured mouse bone marrow macrophages: influence of liposome composition and size." Biochimica et Biophysica Acta (BBA)-Biomembranes 1061(1): 56-64.

Almeida, C., A. Madeira, J. Marto, A. Graça, P. Pinto and H. Ribeiro (2019). "Monfortinho Thermal Water-Based Creams: Effects on Skin Hydration, Psoriasis, and Eczema in Adults." Cosmetics 6(3): 56.

Alves, N. J., W. Cusick, J. F. Stefanick, J. D. Ashley, M. W. Handlogten and B. Bilgicer (2013). "Functionalized liposome purification via liposome extruder purification (LEP)." Analyst 138(17): 4746-4751.

Araujo, A. R., T. Paiva, M. P. Ribeiro and P. Coutinho (2015). Innovation in Thermalism: An Example in Beira Interior Region of Portugal. Health and Wellness Tourism, Springer: 165-180.

Barone, A., M. C. Cristiano, F. Cilurzo, M. Locatelli, D. Iannotta, L. Di Marzio, C. Celia and D. Paolino (2020). "Ammonium glycyrrhizate skin delivery from ultradeformable liposomes: a novel use as an anti-inflammatory agent in topical drug delivery." Colloids and Surfaces B: Biointerfaces: 111152 .

Betz, G., A. Aeppli, N. Menshutina and H. Leuenberger (2005). "In vivo comparison of various liposome formulations for cosmetic application." International journal of pharmaceutics 296(1-2): 44-54.

Blattner, C. M., G. Coman, N. R. Blickenstaff and H. I. Maibach (2014). "Percutaneous absorption of water in skin: a review." Reviews on environmental health 29(3): 175-180. 
Boughlali, M. (2003). "Thermalisme et thalassothérapie en Algérie." Press. Therm. Climat 140: 161-165.

Bruneau, F., D. Bernard, N. Ragueneau and C. Montastier (1996). "Effect of Vichy water on catalase activity in the stratum corneum." International journal of cosmetic science 18(6): 269277.

Cadi, R., J. Beani, S. Belanger, M. Richard and A. Richard (1991). "Effet protecteur de l'application percutanée d'eau thermale Roche-Posay vis-à-vis de la peroxydation lipidique et de la carcinogénèse cutanée induites par les UVB." Les Nouvelles dermatologiques 10(3): 266-272. Carbajo, J. M. and F. Maraver (2018). "Salt water and skin interactions: new lines of evidence." International journal of biometeorology 62(8): 1345-1360.

Castex-Rizzi, N., M. Charveron and C. Merial-Kieny (2011). "Inhibition of TNF-alpha inducedadhesion molecules by Avène Thermal Spring Water in human endothelial cells." Journal of the European Academy of Dermatology and Venereology 25: 6-11.

Celerier, P., P. Litoux, B. Dreno and A. Richard (1995). "Modulatory effects of selenium and strontium salts on keratinocyte-derived inflammatory cytokines." Archives of dermatological research 287(7): 680-682.

Chenaker, H., B. Houha and V. Vincent (2018). "Hydrogeochemistry and geothermometry of thermal water from north-eastern Algeria." Geothermics 75: 137-145.

Ciobanu, M., B. Heurtault, P. Schultz, C. Ruhlmann, C. Muller and B. Frisch (2007). "Layersome: development and optimization of stable liposomes as drug delivery system." International journal of pharmaceutics 344(1-2): 154-157.

Crommelin, D. J. (1984). "Influence of lipid composition and ionic strength on the physical stability of liposomes." Journal of pharmaceutical sciences 73(11): 1559-1563.

Dal Pra, I., A. Chiarini, R. Pacchiana, G. Zumiani, M. Zanoni and U. Armato (2007). "Comano's (Trentino) thermal water interferes with tumour necrosis factor- $\alpha$ expression and interleukin-8 production and secretion by cultured human psoriatic keratinocytes: Yet other mechanisms of its anti-psoriatic action." International journal of molecular medicine 19(3): 373-379.

Elsayed, M. M., O. Y. Abdallah, V. F. Naggar and N. M. Khalafallah (2006). "Deformable liposomes and ethosomes: mechanism of enhanced skin delivery." International journal of pharmaceutics 322(1-2): 60-66.

Fabre, P., H. Cousse, G. Mouzin and M.-T. Trebosc (1992). Liposomes d'eaux thermales stabilises dans un gel d'adn, Google Patents.

Fan, C., X. Li, Y. Zhou, Y. Zhao, S. Ma, W. Li, Y. Liu and G. Li (2013). "Enhanced topical delivery of tetrandrine by ethosomes for treatment of arthritis." BioMed research international 2013.

Ghersetich, I. and T. M. Lotti (1996). "Immunologic aspects: immunology of mineral water spas." Clinics in dermatology 14(6): 563-566.

Hardy, A., C. Seguin, A. Brion, P. Lavalle, P. Schaaf, S. Fournel, L. Bourel-Bonnet, B. t. Frisch and M. De Giorgi (2018). " $\beta$-Cyclodextrin-functionalized chitosan/alginate compact polyelectrolyte complexes (CoPECs) as functional biomaterials with anti-inflammatory properties." ACS applied materials \& interfaces 10(35): 29347-29356.

Hercogova, J., E. Stanghellini, E. Tsoureli-Nikita and G. Menchini (2002). "Inhibitory effects of Leopoldine spa water on inflammation caused by sodium lauryl sulphate." Journal of the European Academy of Dermatology and Venereology 16(3): 263-266.

Heurtault, B., P. Saulnier, B. Pech, J.-E. Proust and J.-P. Benoit (2003). "Physico-chemical stability of colloidal lipid particles." Biomaterials 24(23): 4283-4300. 
Joly, F., J.-E. Branka and L. Lefeuvre (2014). "Thermal water from Uriage-les-Bains exerts DNA protection, induction of catalase activity and Claudin-6 expression on UV irradiated human skin in addition to its own antioxidant properties." Journal of Cosmetics, Dermatological Sciences and Applications 2014.

Joly, F., L. Galoppin, P. Bordat, H. Cousse and E. Neuzil (2000). "Calcium and bicarbonate ions mediate the inhibition of mast cell histamine release by Avene spa water." Fundamental \& clinical pharmacology 14(6): 611-613.

Kakhi, Z., B. Frisch, B. Heurtault and F. Pons (2016). "Liposomal constructs for antitumoral vaccination by the nasal route." Biochimie 130: 14-22.

Karagülle, M. Z., M. Karagülle, S. Kılıç, H. Sevinç, C. Dündar and M. Türkoğlu (2018). "In vitro evaluation of natural thermal mineral waters in human keratinocyte cells: a preliminary study." International journal of biometeorology 62(9): 1657-1661.

Klein, M. E., S. Mauch, M. Rieckmann, D. G. Martínez, G. Hause, M. Noutsias, U. Hofmann, H. Lucas, A. Meister and G. Ramos (2020). "Phosphatidylserine (PS) and phosphatidylglycerol (PG) nanodispersions as potential anti-inflammatory therapeutics: Comparison of in vitro activity and impact of pegylation." Nanomedicine: Nanotechnology, Biology and Medicine 23: 102096.

Kolter, T. and K. Sandhoff (2010). "Lysosomal degradation of membrane lipids." FEBS letters 584(9): 1700-1712.

Kulkarni, S., G. Betageri and M. Singh (1995). "Factors affecting microencapsulation of drugs in liposomes." Journal of microencapsulation 12(3): 229-246.

Largeau, V. (1881). Le Sahara algérien: les déserts de l'Erg, Hachette.

Laugier, J.-P., A. Ribier, A. Richard and J.-P. Brissonnet (1993). Composition pour traitement topique contenant des vésicules lipidiques encapsulant au moins une eau minérale, Google Patents.

Le, C. T. M., A. Houri, N. Balage, B. J. Smith and A. Mechler (2019). "Interaction of small ionic species with phospholipid membranes: The role of metal coordination." Frontiers in Materials 5: 80.

Lee, H.-P., Y.-J. Choi, K.-A. Cho, S.-Y. Woo, S.-T. Yun, J. T. Lee, H. J. Kim, K.-H. Lee and J.W. Kim (2012). "Effect of spa spring water on cytokine expression in human keratinocyte HaCaT cells and on differentiation of CD4+ T cells." Annals of dermatology 24(3): 324-336.

Liu, D., H. Hu, Z. Lin, D. Chen, Y. Zhu, S. Hou and X. Shi (2013). "Quercetin deformable liposome: preparation and efficacy against ultraviolet B induced skin damages in vitro and in vivo." Journal of Photochemistry and Photobiology B: Biology 127: 8-17.

Liu, H., P. Sidiropoulos, G. Song, L. J. Pagliari, M. J. Birrer, B. Stein, J. Anrather and R. M. Pope (2000). "TNF- $\alpha$ gene expression in macrophages: regulation by NF- $\kappa \mathrm{B}$ is independent of cJun or C/EBP $\beta . "$ The Journal of Immunology 164(8): 4277-4285.

MacMicking, J., Q.-W. Xie and C. Nathan (1997). "Nitric oxide and macrophage function." Annual review of immunology 15(1): 323-350.

Malissen, B., S. Tamoutounour and S. Henri (2014). "The origins and functions of dendritic cells and macrophages in the skin." Nature Reviews Immunology 14(6): 417-428.

Manosroi, A., W. Witkittilak, R. Chutoprapat, H. Todo, K. Sugibayashi, W. Manosroi and J. Manosroi (2010). "Moisture enhancement of niosomes entrapped with mineral water in pig ear skin." Chiang Mai J. Sci 38(1): 126-138.

Manosroi, A., P. Wongtrakul, J. Manosroi, H. Sakai, F. Sugawara, M. Yuasa and M. Abe (2003). "Characterization of vesicles prepared with various non-ionic surfactants mixed with cholesterol." Colloids and Surfaces B: Biointerfaces 30(1-2): 129-138. 
Maxwell, T., K.-S. Lee, S.-Y. Chun and K.-S. Nam (2017). "Mineral-balanced deep sea water enhances the inhibitory effects of chitosan oligosaccharide on atopic dermatitis-like inflammatory response." Biotechnology and Bioprocess Engineering 22(2): 120-128.

McLAUGHLIN, S., N. Mulrine, T. Gresalfi, G. Vaio and A. McLAUGHLIN (1981). "Adsorption of divalent cations to bilayer membranes containing phosphatidylserine." The Journal of general physiology 77(4): 445-473.

Merial-Kieny, C., N. Castex-Rizzi, B. Selas, S. Mery and D. Guerrero (2011). "Avène Thermal Spring Water: an active component with specific properties." Journal of the European Academy of Dermatology and Venereology 25: 2-5.

Merial-Kieny, C., X. Mengual, D. Guerrero and V. Sibaud (2011). "Clinical efficacy of Avène hydrotherapy measured in a large cohort of more than 10,000 atopic or psoriatic patients." Journal of the European Academy of Dermatology and Venereology 25: 30-34.

Nasermoaddeli, A. and S. Kagamimori (2005). "Balneotherapy in medicine: a review." Environmental Health and Preventive Medicine 10(4): 171-179.

Nathan, C. and Q.-w. Xie (1994). "Nitric oxide synthases: roles, tolls, and controls." Cell 78(6): 915-918.

Nunes, F., M. Rodrigues, M. P. Ribeiro, E. Ugazio, R. Cavalli, O. Abollino, P. Coutinho and A. R. Araujo (2019). "Incorporation of Cró thermal water in a dermocosmetic formulation: cytotoxicity effects, characterization and stability studies and efficacy evaluation." International journal of cosmetic science 41(6): 604-612.

Nunes, S. and B. Tamura (2012). "Avaliação físico-química e biológica da eficácia de uma água mineral lipossomada na pele." Surgical \& Cosmetic Dermatology 4(1): 17-21.

Pasparakis, M., I. Haase and F. O. Nestle (2014). "Mechanisms regulating skin immunity and inflammation." Nature reviews immunology 14(5): 289-301.

Prasad, A. S. (2014). "Zinc: an antioxidant and anti-inflammatory agent: role of zinc in degenerative disorders of aging." Journal of Trace Elements in Medicine and Biology 28(4): 364371.

Ribeiro, M., A. Araújo and P. Coutinho (2015). "Production of mineral delivery system with thermal mineral water for dermatologic application."

Richard, M., P. Guiraud, J. Arnaud, R. Cadi and A. Monjo (1990). "Pouvoir antioxydant d'une eau thermale séléniée sur des fibroblastes cutanés humains diploïdes." Les Nouvelles dermatologiques 9: 257-261.

Seite, S. (2013). "Thermal waters as cosmeceuticals: La Roche-Posay thermal spring water example." Clinical, cosmetic and investigational dermatology 6: 23.

Shukla, M. and S. Arya (2018). "Determination of Chloride ion (Cl-) concentration in ganga river water by Mohr method at Kanpur, India." Green Chemistry \& Technology Letters 4(1): 6-8.

Tacheau, C., F. Weisgerber, D. Fagot, P. Bastien, M. Verdier, M. Liboutet, G. Sore and B. Bernard (2018). "Vichy Thermal Spring Water (VTSW), a cosmetic ingredient of potential interest in the frame of skin ageing exposome: an in vitro study." International journal of cosmetic science 40(4): 377-387.

Tamura, B., D. M. Pedroso and S. Nunes (2012). "Evaluation of the effects of Serra do Japi's liposomal mineral water on the skin." Journal of the American Academy of Dermatology 66(4).

Tan, J., J. Spada, C. Orlandi, M. Kerscher, M. Anfilova, S. Abdulla, A. Floriánová, K. Preisz, A. Altmayer and C. Delva (2020). "Vichy mineralizing water with hyaluronic acid is effective and well tolerated as an adjunct to the management of various dermatoses and after esthetic procedures." Journal of Cosmetic Dermatology 19(3): 682-688. 
Treede, I., A. Braun, R. Sparla, M. Kühnel, T. Giese, J. R. Turner, E. Anes, H. Kulaksiz, J. Füllekrug and W. Stremmel (2007). "Anti-inflammatory effects of phosphatidylcholine." Journal of Biological Chemistry 282(37): 27155-27164.

Tsoureli-Nikita, E., G. Menchini, I. Ghersetich and J. Hercogova (2002). "Alternative treatment of psoriasis with balneotherapy using Leopoldine spa water." Journal of the European Academy of Dermatology and Venereology 16(3): 260-262.

Verdy, C., J.-E. Branka and L. Lefeuvre (2012). "Modulation of sodium-dependent transporters expression in normal human keratinocytes by a sodium rich isotonic thermal water."

Verma, D., S. Verma, G. Blume and A. Fahr (2003). "Liposomes increase skin penetration of entrapped and non-entrapped hydrophilic substances into human skin: a skin penetration and confocal laser scanning microscopy study." European Journal of Pharmaceutics and Biopharmaceutics 55(3): 271-277.

Wang, X., C. J. Swing, T. Feng, S. Xia, J. Yu and X. Zhang (2020). "Effects of environmental pH and ionic strength on the physical stability of cinnamaldehyde-loaded liposomes." Journal of Dispersion Science and Technology 41(10): 1568-1575.

Wollenberg, A., A. Richard and T. Bieber (1992). "In vitro effect of the thermal water from La Roche-Posay on the stimulatory capacity of epidermal Langerhans cells." Eur J Dermatol 2: 128129.

Xie, Q.-w., H. J. Cho, J. Calaycay, R. A. Mumford, K. M. Swiderek, T. D. Lee, A. Ding, T. Troso and C. Nathan (1992). "Cloning and characterization of inducible nitric oxide synthase from mouse macrophages." Science 256(5054): 225-228.

Yamaguchi, T., M. Nomura, T. Matsuoka and S. Koda (2009). "Effects of frequency and power of ultrasound on the size reduction of liposome." Chemistry and physics of lipids 160(1): 58-62. 